\title{
Assistência de enfermagem na manutenção do acesso vascular arteriovenoso de pacientes renais crônicos em hemodiálise: uma revisão narrativa
}

Nursing care in the maintenance of arteriovenous vascular access in chronic renal patients undergoing hemodyalysis: an narrative review

Atención de enfermería en el mantenimiento del acceso vascular arteriovenoso de los pacientes renales crónicos en hemodiálisis: una revisión narrativa

Rodrigo Santos da Silva ${ }^{1}$, Shirlley Sayonara Bezerra de Melo Torres ${ }^{2}$, Angélica de Godoy Torres Lima $^{3 *}$.

\section{RESUMO}

Objetivo: Descrever os cuidados de enfermagem para a manutenção da fístula arteriovenosa em pacientes renais crônicos em terapia hemodialítica. Revisão bibliográfica: Para realizar a hemodiálise, o paciente necessita de um acesso vascular como uma fístula arteriovenosa, cateter venoso de duplo lúmen ou enxerto. A fístula arteriovenosa se tornou para o paciente em tratamento dialítico, um meio importante para a realização do tratamento, considerada um método seguro que proporciona conforto e autonomia em relação ao cateter venoso de duplo lúmen. Para minimizar as complicações com a fístula arteriovenosa, é necessário a colaboração entre os profissionais de saúde, desde nefrologista, cirurgião vascular, enfermeiros e o próprio doente. Considerações finais: É fundamental para o enfermeiro desenvolver habilidades para avaliar e diagnosticar todas as complicações que possam ocorrer com a fístula arteriovenosa, sendo possível através da aplicação da sistematização da assistência de enfermagem com esses pacientes.

Palavras-chave: Insuficiência renal crônica, Fístula arteriovenosa, Cuidados de enfermagem.

\begin{abstract}
Objective: To describe the nursing care in the maintenance of arteriovenous fistula of chronic renal patients undergoing hemodialysis therapy. Bibliographic review: To perform hemodialysis, the patient needs a vascular access such as an arteriovenous fistula, double-lumen venous catheter or graft. The arteriovenous fistula has become a dialysis treatment for the patient, an important means for the treatment, considered a safe method that provides comfort and autonomy in relation to the double lumen venous catheter. To minimize complications with arteriovenous fistula, collaboration between health professionals, from nephrologists, vascular surgeons, nurses and the patient himself, is necessary. Conclusion: It is essential for nurses to develop skills to assess and diagnose all complications that may occur with arteriovenous fistula, being possible through the application of systematized nursing care for these patients.
\end{abstract}

Keywords: Renal insufficiency chronic, Arteriovenous fistula; Nursing care.

\section{RESUMEN}

Objetivo: Describir la atención de enfermería para el mantenimiento de la fístula arteriovenosa de los pacientes renales crónicos en la terapia de hemodiálisis. Revisión bibliográfica: para realizar la hemodiálisis,

\footnotetext{
${ }^{1}$ Faculdade do Instituto de Desenvolvimento Educacional (Faculdade IDE), Recife-PE.

${ }^{2}$ Centro de Formação, Pós-graduação e Pesquisa em saúde (CEFAPP), Recife-PE.

3 Instituto Federal de Educação, Ciência e Tecnologia de Pernambuco campus Belo Jardim (IFPE), Belo

Jardim-PE. * E-mail: angelica.godoy@belojardim.ifpe.edu
} 
el paciente necesita un acceso vascular como una fístula arteriovenosa, un catéter venoso de doble luz o un injerto. La fístula arteriovenosa se ha convertido en un tratamiento de diálisis para el paciente, un medio importante para el tratamiento, considerado un método seguro que proporciona comodidad y autonomía en relación con el catéter venoso de doble luz. Para minimizar las complicaciones con la fístula arteriovenosa, es necesaria la colaboración entre profesionales de la salud, de nefrólogos, cirujanos vasculares, enfermeras y el propio paciente. Consideraciones finales: Es esencial que las enfermeras desarrollen habilidades para evaluar y diagnosticar todas las complicaciones que pueden ocurrir con la fístula arteriovenosa, siendo posible mediante la aplicación de cuidados de enfermería sistematizados para estos pacientes.

Palabras clave: Insuficiencia renal crónica, Fístula arteriovenosa, Atención de enfermería.

\section{INTRODUÇÃO}

A doença renal crônica (DRC) caracteriza-se pela perda progressiva e irreversível da função renal. Sua definição se dá a partir de dois critérios, que podem aparecer em conjunto ou isoladamente. São eles: anormalidades estruturais e/ou funcionais do rim por um período maior ou igual há três meses e lesões estruturais renais e/ou taxa de filtração glomerular menor que $60 \mathrm{ml} / \mathrm{min} . / 1,73 \mathrm{~m}^{2}$; nesse último, com ou sem lesão do parênquima renal (RIBEIRO KRA, 2016; SILVA CT e CHRISTOVAM BP, 2015).

Na Europa e nos Estudos Unidos da América, o diabetes mellitus é a principal causa da doença renal crônica e está associado à quase metade de novos casos de DRC. A hipertensão arterial, embora não seja a mais importante, é diagnóstico de base frequente em países como EUA, França e Itália (SILVA CT e CHRISTOVAM BP, 2015).

De acordo com Sesso RC, et al. (2014) através de dados da Sociedade Brasileira de Nefrologia (SBN), no Brasil, as principais doenças de base da DRC, entre os pacientes em programa dialítico, são respectivamente: a nefropatia hipertensiva, o diabetes mellitus, as glomerulonefrites e rins policísticos. O Brasil tem o terceiro maior contingente de pacientes em hemodiálise (HD) no mundo. Em 2012, o número total estimado de pacientes em diálise no país era de 97.586, distribuídos em 651 unidades com programa crônico de diálise.

Segundo Guimarães GL, et al. (2017) essa modalidade exige aparato tecnológico envolvendo máquinas, insumos, profissionais treinados e a confecção de um acesso vascular (AV) no paciente. A fístula arteriovenosa (FAV) é considerada como acesso "padrão ouro" da hemodiálise. Quando o paciente necessita dialisar antes da fistula estar pronta, o procedimento é realizado através de cateter central de duplo lúmen (HORTA HHL e LOPES ML, 2017).

O AV constitui um requisito indispensável para a realização e sucesso da HD e para a melhora na qualidade de vida do doente em diálise. Nesta perspectiva, o acesso à circulação para tratamento dialítico de repetição é um dos aspectos mais importantes em HD crônica em doentes com doença renal crônica terminal (DRCT), até porque as complicações relacionadas com o acesso vascular para HD são responsáveis por $25 \%$ dos internamentos e por $50 \%$ dos custos hospitalares neste grupo de doentes (MARTINS PTC, 2015). Após a construção do $\mathrm{AV}$, é necessário que os centros de diálise utilizem programas de melhoria contínua da qualidade para detectar os $\mathrm{AV}$ em risco, rastrear taxas de complicações do AV e implementar procedimentos que maximizem a longevidade do AV (ROCHA R, 2017).

Com a finalidade de manter a segurança ao paciente, qualidade da assistência e autonomia profissional do enfermeiro, a Sistematização da Assistência de Enfermagem (SAE) vem sendo praticada na assistência em hemodiálise, visto que essa organiza o trabalho quanto ao método, recursos humanos e instrumentos, além de viabilizar a operacionalização do Processo de Enfermagem (PE). Esse instrumento metodológico gera visibilidade e reconhecimento social à profissão através da orientação do cuidado e da documentação da prática assistencial (GUIMARÃES GL, et al., 2016).

Devido à complexidade de cuidados exigidos do profissional para com os pacientes em hemodiálise, cujas condições clínicas mudam constantemente e observando que as FAV, são manipuladas pela equipe de enfermagem sob a supervisão dos enfermeiros acredita-se que intervenções que visam resultados clínicos satisfatórios, a partir da competência técnico-científica e humanística presentes no cuidado de enfermagem, poder-se-á melhorar e manter o cuidado com a FAV, proporcionando segurança ao paciente e autonomia ao enfermeiro (GUIMARÃES GL, et al., 2016). 
Diante do exposto, é imprescindível que o enfermeiro esteja presente nas sessões de hemodiálise coordenando a equipe e identificando as necessidades particulares de cada paciente. Desta forma, colabora para o crescimento e desenvolvimento expressivo da assistência de enfermagem em nefrologia de excelência por meio dos processos estabelecidos pela SAE.

A realização desse estudo justifica-se com o intuito de contribuir para a reflexão do enfermeiro nefrologista quanto a assistência baseada em evidências científicas direcionadas para as boas práticas de manutenção da FAV, visto que a manutenção do acesso vascular em diálise proporciona a esses pacientes aumento da sobrevida e da qualidade de vida. O presente estudo tem por objetivo descrever os cuidados de enfermagem para a manutenção da fístula arteriovenosa de pacientes renais crônicos em terapia hemodialítica através de uma revisão de literatura.

\section{REVISÃO BIBLIOGRÁFICA}

\section{O contexto da Pessoa com Doença Renal Crônica em Hemodiálise.}

A DRC é um problema de saúde pública que tem aumentado de forma significativa sua prevalência e incidência em países com diversas características econômicas. É uma doença lenta e silenciosa que provoca perda total das funções dos rins, tais como de regulação, de excreção e endócrina, levando à necessidade da adoção de terapias renais substitutivas, como a hemodiálise (PAIVA PA et al., 2018).

Segundo Xavier V e Lima CB (2018) a DRC é considerada no atual contexto social por diversos especialistas da área como uma espécie de pandemia. Particularmente no Brasil, o índice de crescimento dessa patologia é avassalador entre a população, exibindo um péssimo prognóstico. Esta situação vem acarretando sérios problemas à saúde da população, com sofrimento, limitações e perdas, o que se agrava com os custos do tratamento que são caríssimos. Apesar dos avanços em conhecimento científico específico, em tecnologias e equipamentos, o número de pessoas vítimas dessa patologia progride em escala ascendente em nível global. A dimensão do dilema é tão preocupante que nos dias atuais esta enfermidade vem sendo considerada como uma questão de saúde pública.

Ressalte-se que o Censo 2012 da SBN contabilizou um quantitativo de 10 milhões de pessoas acometidas por algum tipo de distúrbio renal, sendo que cerca de $70 \%$ ignoram a sua existência. No Brasil, foram realizados 91.475 procedimentos de hemodiálise no ano de 2008, em 2012, 118.847. Para o ano 2017, estimou-se um aumento de $24,8 \%$ na realização desse procedimento (SESSO RC, et al., 2014; XAVIER V e LIMA CB, 2017; PAIVA PA, et al, 2018).

Ao se deparar com essa necessidade o paciente passa por um processo de rejeição e de aceitação, que interfere no seu estado emocional e psicológico, gerando algumas consequências na forma de enfrentar a doença e o tratamento (PAIVA PA, et al., 2018).

Quando intensamente sintomáticos, os portadores de lesão renal com insuficiência renal terminal, tem a necessidade de realizar alguma modalidade de terapia renal substitutiva (TRS): diálise peritoneal, hemodiálise ou o transplante renal.

A hemodiálise é um tratamento dialítico em que o sangue é bombeado por meio de um AV, permitindo um fluxo sanguíneo extracorpóreo em direção a um filtro artificial composto por uma membrana semipermeável e que extrai do sangue toxinas, resíduos nitrogenados e água em excesso, devolvendo, em seguida, o sangue limpo ao paciente.

Habitualmente, ela é realizada de três a quatro vezes por semana e com durações variadas de três a quatro horas em cada sessão, consistindo no recurso mais utilizado por toda a vida na DRC ou até o paciente submeter-se a um transplante renal bem-sucedido (NETO JMR, et al., 2016).

O transplante renal é considerado o tratamento mais adequado para a insuficiência renal crônica; entretanto, é necessário selecionar entre os portadores de insuficiência renal crônica, aqueles que estão de acordo com os critérios, levando-se em consideração o aspecto técnico-cirúrgico, sobrevida e recidiva da doença (SANTOS WN et al., 2016). 
De acordo com Horta HHL e Lopes ML (2017) o paciente com DRC convive diariamente com uma doença incurável e de longa duração. Com a evolução da doença, surgem complicações a curto e longo prazo que provocam limitações e alterações que repercutem na vida do doente e de seus familiares.

Para melhor adaptação, o diagnóstico precoce da DRC é fundamental, uma vez que a descoberta tardia se deve à falta de conhecimento da população acerca da prevenção e também da pouca informação oferecida pelos profissionais de saúde na atenção em saúde. Sendo assim, o trabalho do enfermeiro no processo de educação nas unidades de saúde do Sistema Único de Saúde (SUS) é de extrema importância devido a sua proximidade com os usuários, atuando na prevenção de várias doenças crônicas, como a DRC.

Nessa relação, o diálogo é uma peça fundamental de meio educativo que propicia ao indivíduo e sua família uma relação horizontal com o profissional de saúde, na qual são expostas as dúvidas a respeito da enfermidade e do tratamento, ressaltando que a DRC é grave e exige mudança brusca no estilo de vida do indivíduo por ela acometido.

Oportuno se faz evidenciar que, a partir do diagnóstico da patologia intercorrem várias modificações no cotidiano do enfermo. Essas transformações não se limitam apenas a ele, mas também a sua família que se não for bem amparada poderá não suportar as adversidades desencadeadas pela moléstia (XAVIER $V$ e LIMA CB, 2018).

O apoio da enfermagem é primordial para esses pacientes durante o tratamento. Para tanto, é preciso uma perfeita integração e treinamento entre os componentes da equipe, e dela com os demais profissionais de saúde.

O enfermeiro nefrologista deve ser tolerante, solícito e sensível, compartilhando sentimentos, mantendo a humildade e a compaixão, para proporcionar aos pacientes com DRC o conforto necessário para aceitar o tratamento. Assim, ressalta-se a importância da implementação de estratégias que contribuiam para que o paciente se torne cada vez mais participativo e atuante no seu tratamento, possibilitando sua autonomia e estimulando o cuidado de si como uma possibilidade para melhor adesão à hemodiálise e à promoção da qualidade de vida (SANTOS BP, et al., 2017).

\section{A Fístula Arteriovenosa como Acesso em Hemodiálise e o Papel do Enfermeiro Frente aos Cuidados.}

Para realizar a hemodiálise, o paciente necessita de um AV como uma fístula arteriovenosa, cateter venoso de duplo lúmen (CDL) ou enxerto. A FAV se tornou para o paciente em tratamento dialítico um meio importante para a realização do tratamento, considerada um método seguro que proporciona conforto e autonomia em relação ao CDL (NOGUEIRA FLL, et al., 2016).

Santos BP, et al. (2017) narram que a técnica cirúrgica da FAV foi criada por Cimino e Brescia, por meio de uma anastomose interna, sendo um grande achado no tratamento da DRC. Além do mais, a criação da fístula foi importante para o sucesso terapêutico dos pacientes renais crônicos, pois garante um fluxo de sangue maior no local da punção e é um acesso vascular permanente.

A fístula arteriovenosa para hemodiálise (FAVH) é a anastomose entre uma artéria e uma veia superficial, com o objetivo de criar um maior fluxo na veia, gerando uma dilatação venosa com espessamento da parede do vaso, para uma futura punção e utilização na hemodiálise que requer a passagem de altos fluxo de sangue dentro do mesmo. A dilatação desta veia e sua extensão são essenciais para o funcionamento do acesso, porque durante a hemodiálise a veia receberá duas punções simultâneas (PEREIRA OR, et al., 2016).

O processo de maturação da fístula arteriovenosa ainda não está bem determinado, acontecendo em um período que varia entre 4 e 12 semanas. Durante a fase de pós-operatório da FAVH advêm múltiplas alterações nos vasos que foram utilizados para a confecção da fístula. $\mathrm{Na}$ artéria acontece uma dilatação da artéria aferente e aumento do fluxo sanguíneo arterial. Na veia ocorre a dilatação, o espessamento da parede da mesma e o aumento do fluxo sanguíneo ocasionado pela conexão da FAVH (PEREIRA OR, et al., 2016).

Neto JMR, et al. (2016) relatam em seu estudo que quanto a sua localização, elas se classificam em distais, incluindo as FAV radiocefálicas no punho e no antebraço; e proximais, que incluem as braquiocefálica, braquiobasílica superficializada e braquioaxilar ou braquiobraquial em alça com prótese. 
A fistula arteriovenosa vem sendo considerada unanimemente pela comunidade científica como o acesso vascular de excelência para hemodiálise. Este acesso providencia durabilidade superior, menos infeções, trombose e hospitalização em comparação com os cateteres venosos centrais e as próteses. Ainda de acordo com os mesmos autores, para minimizar problemas com o acesso vascular, é necessária uma estreita colaboração entre os profissionais de saúde e os doentes em hemodiálise para potenciar a sua vigilância (SILVA AS, 2017).

Assim, para minimizar as complicações com a FAV, é necessário a colaboração entre os profissionais de saúde, desde nefrologista, cirurgião vascular, enfermeiros e o próprio doente. O enfermeiro na sua relação com a pessoa receptora de cuidados "necessita demostrar competências técnico-científicas, psicossociais e ético-deontológicas para que consiga obter os melhores resultados, que se traduzem pela longevidade da FAV e na melhoria da qualidade de vida da pessoa com DRC" (BARROS AO, 2017).

Barros AO (2017) define o exame físico como a "habilidade que o enfermeiro realiza na sua prática do cuidar, envolvendo os órgãos dos sentidos, a nível da visão (olhos), do tacto (mãos) e da audição (ouvidos), com o objetivo de recolher informações relevantes". Na FAV temos sangue arterial a passar para o sistema venoso que origina uma vibração e som característicos (pulso, frémito e sopro), que podem ser identificados através da palpação e auscultação.

A avaliação do pulso é através da palpação, um pulso suave e compressível é normal. Um pulso anormal é quando o "inflow" é fraco, podendo estar relacionado com um problema arterial ou da anastomose arteriovenosa, ou "inflow" é forte, pode indicar uma estenose venosa.

O frêmito é avaliado através da palpação, sentindo uma sensação de zumbido que vai diminuindo ao longo da veia de drenagem. O sopro é avaliado através da auscultação, deve ser contínuo na sístole e na diástole, sendo mais intenso na anastomose e vai diminuindo de intensidade ao longo da veia de drenagem (BARROS AO, 2017).

Mesmo sendo o padrão-ouro em acesso vascular para a hemodiálise, a FAV é suscetível a várias complicações, tais como: hipofluxosanguíneo, tromboses, aneurismas, infecções, isquemia de mão, edema de mão e sobrecarga cardíaca. Os cuidados adequados podem prevenir grande parte dessas complicações, portanto, o paciente renal crônico precisa ser orientado acerca do autocuidado no período de confecção e no manejo do seu novo acesso vascular, visto que a responsabilidade das ações envolvidas é compartilhada entre a equipe de saúde e o paciente (PEREIRA M, et al., 2017; SOUSA CN, 2012).

No período de maturação da FAV, a assistência de enfermagem tem o objetivo de promover a maior durabilidade da fístula e compreende ações como a manutenção do braço elevado, evitar curativos circunferenciais apertados, realizar a avaliação do fluxo sanguíneo diariamente e fazer exercícios de compressão manual. Além do mais, durante todo o período de utilização da fístula, alguns cuidados devem ser realizados, tais como a compressão adequada para hemostasia após a diálise, poupar a utilização do membro do acesso ao evitar fazer grandes esforços, infusões venosas e medidas de pressão arterial no mesmo (PEREIRA M, et al., 2017).

Independentemente do método de punção da FAV, o procedimento é asséptico. É recomendada a preparação da pele adjacente ao acesso, inicialmente pela higienização da região da FAV com sabão antibacteriano, e então antissepsia do local da punção com solução antisséptica imediatamente antes da punção. A direção da agulha "arterial" pode ser anterógrada (na direção do fluxo sanguíneo) ou retrógrada (contra a direção do fluxo sanguíneo).

A decisão da direção da punção "arterial" é influenciada pelo espaço de punção que a FAV dispõe. Após a punção, deve-se fixar a agulha no mesmo ângulo ou ângulo similar da inserção para evitar traumas na parede vascular. Ao final do tratamento, as agulhas devem ser retiradas no mesmo ângulo de inserção e não se deve pressionar o local de punção antes da remoção total da agulha para evitar traumas à camada íntima do vaso e complicações como infiltrações (RODRIGUES JG, 2018).

É recomendado que no início da utilização da FAV sejam aplicadas agulhas de menor calibre e fluxo sanguíneo como a $17 \mathrm{G}$ e $16 \mathrm{G}$ que oferecem de 250 a $350 \mathrm{~mL} / \mathrm{min}$ respectivamente. Já para FAV madura e 
calibrosa, é recomendado o uso de agulhas de maior calibre e fluxo de sangue como a $15 G$ que oferece fluxo de sangue de até $450 \mathrm{~mL} / \mathrm{min}$. A escolha do calibre da agulha também dependerá da necessidade da adequação de qualidade dialítica do paciente pela equipe de saúde (RODRIGUES JG, 2018).

Sousa CN (2012) desenvolveu de uma estrutura conceitual direcionada para a prática do cuidar da pessoa com FAV, a qual integra conceitos que se relacionam entre si, cujos objetivos concernem à capacitação da pessoa para o autocuidado; à identificação de complicações e realização do tratamento. Nesse modelo de SAE há a descrição e orientação dos cuidados a serem desenvolvidos pelos enfermeiros no PE, permitindo a sistematização das intervenções autônomas de enfermagem.

Essa estrutura colabora para o desenvolvimento de competências cognitivas e comportamentais que permitem ao enfermeiro nefrologista, ser capaz de avaliar e interpretar eficazmente os dados objetivos da FAV e do monitor, articulando-os com os dados subjetivos adquiridos pela interação com a pessoa; possibilitando que os cuidados de enfermagem sejam expressos sucessivamente num nível elevado de qualidade (SOUSA CN, 2012).

O indivíduo deve ser dotado de competências para saber cuidar da sua FAV, tanto no momento que antecede a construção do acesso vascular quanto após a utilização deste. A ação de enfermagem baseia-se em intervenções terapêuticas que se antecipam a fim de promover o início do percurso, a manutenção/acompanhamento e de finalização da transição mais saudável possível. Portanto, é fundamental que o cliente desenvolva conhecimentos de autocuidado antes da construção da FAV que visem preservar a sua rede venosa, de forma a manter um patrimônio vascular íntegro, limitando assim a morbilidade e os custos associados aos acessos vasculares (SILVA AS, 2017; PEREIRA HDR, 2018; SOUSA CN, 2012).

Em relação aos cuidados interdialíticos é essencial elucidar ao paciente como proceder no domicílio. A prioridade do enfermeiro é ensinar às pessoas/cuidadores a importância da observação diária do acesso vascular, para que estes sejam capazes de reconhecer sinais de complicações, tais como: rubor, calor ao toque, dor, exsudado e edema no braço; realizar cotidianamente a palpação sobre a FAV para sentir vibração/sensação de zumbido rítmico (frêmito) e verificar se a região está dolorosa ou mais quente que o habitual (PEREIRA HDR, 2018; SILVA AS, 2017).

Na zona da FAV deve-se garantir a manutenção de uma temperatura constante, evitando o calor excessivo (saunas e banhos a vapor), já que este induz a vasodilatação e a redução da tensão arterial com consequentes redução do fluxo sanguíneo. A vasoconstrição pelo frio excessivo também deve ser evitada, porque esta induz a redução do fluxo sanguíneo dos membros, especialmente no membro da FAV a fim de evitar hipoperfusão (PEREIRA HDR, 2018).

Os enfermeiros devem orientar aos pacientes a entrar em contato com o centro de hemodiálise, assim que possível, em caso de complicações como quando o frêmito da FAV para ou diminui bruscamente; alterações na aparência da pele ou do braço da FAV; casos de traumatismo diretamente na FAV ou no membro da FAV.

Os pacientes devem ser ensinados a evitar transportar objetos pesados com o membro da FAV além de alertar outros profissionais de saúde para não aferirem a pressão arterial ou realizar qualquer tipo procedimento de coleta de sangue, injeção ou cateterização no membro do AV (SILVA AS, 2017; PEREIRA HDR, 2018).

\section{CONSIDERAÇÕES FINAIS}

Devido à natureza do seu ofício, os enfermeiros são os profissionais de saúde que atuam diretamente com a FAV e manipulam o acesso vascular da pessoa com DRC em tratamento hemodialítico. Portanto, é fundamental para estes profissionais desenvolver habilidades para avaliar e diagnosticar todas as complicações que possam ocorrer com a FAV. A aplicação da SAE na prática do cuidado à pessoa com FAV promove o desenvolvimento de competências cognitivas e habilidades que capacitam os enfermeiros a identificar e diagnosticar precocemente alterações no funcionamento da FAV, e, consequentemente, permite um aumento na sobrevida desse tipo de acesso vascular, melhorando o bem-estar e qualidade de vida dos pacientes. 


\section{REFERÊNCIAS}

1. BARROS AO. Intervenção do Enfermeiro Especialista em Enfermagem Nefrológica na Manutenção da Fístula Arteriovenosa à Pessoa Hemodialisada. Dissertação (Mestrado em Enfermagem Médico Cirúrgica na vertente Nefrológica) - Escola Superior de enfermagem de Lisboa, Lisboa, 2017; 140 p.

2. GUIMARÃES GL, et al. Intervenções de Enfermagem no Paciente em Hemodiálise por Cateter Venoso Central. Rev enferm UFPE on line, 2017; 11(3):1127-35.

3. GUIMARÃES GL, et al. Contribuição da Teoria de Horta para Crítica dos Diagnósticos de Enfermagem no Paciente em Hemodiálise. Rev enferm UFPE on line, 2016; 10(2): 554-61.

4. HORTA HHL, LOPES ML. Complicações Decorrentes do Tratamento Dialítico: Contribuição do Enfermeiro no Cuidado e Educação ao Paciente. Revista Enfermagem Contemporânea, 2017; 6(2): 221-227.

5. MARTINS PTC. Cuidados de Enfermagem para a Manutenção dos Acessos Vasculares de Hemodiálise no Serviço de Urgência. Dissertação (Mestrado em Enfermagem Médico Cirúrgica na vertente Nefrológica). Escola Superior de enfermagem de Lisboa, Lisboa, 2015; 248 p.

6. NETO JMR, et al. Fístula Arteriovenosa na Perspectiva de Pacientes Renais Crônicos. Enferm Foco, 2016; 7(1): 3741.

7. NOGUEIRA FLL, et al. Percepção do Paciente Renal Crônico Acerca dos Cuidados com Acessos para Hemodiálise. Cogitare Enferm, 2016; 21(3): 01-08.

8. PAIVA PA, et al. Incidência de Infecções da Corrente Sanguínea em Pacientes Nefropatas. Rev Aten Saúde, 2018; 16(55): 72-80.

9. PEREIRA HDR. Autocuidado com a Fístula Arteriovenosa da Pessoa em Programa Regular de Hemodiálise. Dissertação (IV CURSO DE MESTRADO EM ENFERMAGEM MÉDICO-CIRÚRGICA) - Escola Superior de Saúde. Instituto Politécnico, Viana do Castelo, 2018; 142 p.

10. PEREIRA M, et al. Acesso Vascular life-saving na Exaustão do Capital Vascular: Experiência de um Centro com Cateteres Intra-Auriculares para Hemodiálise. J Bras Nefrol, 2017; 39(1): 36-41

11. PEREIRA OR, et al. Avaliação do Tempo de Maturação das Fístulas Rádio-Cefálicas para Hemodiálise. Arq Catarin Med, 2016; 45(2): 02-10.

12. RIBEIRO KRA. Cuidados de Enfermagem aos Pacientes com Insuficiência Renal Crônica no Ambiente Hospitalar. Revista Recien, 2016; 6(18):26-35.

13. ROCHA R. O Papel do Enfermeiro Especialista na Equipa de Coordenação do Acesso Vascular Arteriovenoso - De Iniciado a Perito. Dissertação (Mestrado em Enfermagem Médico Cirúrgica na vertente Nefrológica). Escola Superior de enfermagem de Lisboa, Lisboa, 2017; 308 p.

14. RODRIGUES JG. Punção de Fístula Arteriovenosa de Pacientes em Hemodiálise: Evidências para a Enfermagem. Dissertação (Mestrado A enfermagem no cuidado à Saúde Humana) - Faculdade de Enfermagem (FEN), Programa de Pós-Graduação em Enfermagem. Universidade Federal de Goiás, Goiânia, 2018; 71 p.

15. SANTOS BP, et al. Doença Renal Crônica: Relação dos Pacientes com a Hemodiálise. ABCS Health Sci, $2017 ; 42$ (1): 8-14.

16. SANTOS WN, et al. Atuação do Enfermeiro nas Complicações Decorrentes do Transplante Renal: uma Revisão de Literatura. Revista UNINGÁ Review, 2016; 25 (1):131-142.

17. SESSO RC, et al. Relatório do Censo Brasileiro de Diálise Crônica 2012. Braz. J Nephrol, $2014 ; 36$ (1): $48-53$.

18. SILVA AS. Autocuidado na Manutenção do Acesso Vascular para Hemodiálise. Dissertação (Mestrado em Enfermagem Médico Cirúrgica na vertente Nefrológica). Escola Superior de enfermagem de Lisboa, Lisboa, $2017 ; 65$ p.

19. SILVA CT, CHRISTOVAM BP. As Ações de Gerência do Cuidado em Serviço de Hemodiálise: Revisão Integrativa. Rev enferm UFPE on line, 2015; 9 (8): 8890-8898.

20. SOUSA CN. Cuidar da pessoa com fístula arteriovenosa: modelo para a melhoria continua. Rev. Port. Saúde Pública, 2012; 30(1):11-17.

21. XAVIER V, LIMA CB. Tratamento da Doença Renal Crônica: Abordando as Contribuições da Teoria do Autocuida,do. Temas em Saúde, 2018; 18(1): 305-323. 